\title{
Utilization of Electric Vehicles Connected to Distribution Substations for Peak Shaving of Utility Network Loads
}

\author{
Marek Mägi (Doctoral student, Tallinn University of Technology)
}

\begin{abstract}
Use of modern electric vehicles and their effective integration into power grids depends on the technologies applied around distribution substations. Distribution substations equipped with energy storing and V2G capability enable peak load shaving and demand response, which will reduce the need to make new investments into building new power sources or power grids to meet peak demand. This paper presents a distribution substation topology for utilizing electric vehicles as energy resource units for peak shaving of utility network loads. The topology allows bidirectional energy exchange among electric vehicles, battery pack energy storage devices and utility networks. The substation acts as a service provider in a microgrid. Functions of a microgrid application were simulated with MATLAB. The evaluation of the results has shown that electric vehicles can be effectively utilized for peak shaving of utility network loads. The results of the modelling and simulation were used for the development of a microgrid prototype. Assessment of capacities of electric vehicles showed that electric vehicles can provide short term support for the utility network.
\end{abstract}

Keywords - AC-DC power converters, bidirectional power flow, DC-DC power converters, electric vehicles, substations.

\section{INTRODUCTION}

Smart Grids and microgrids have attracted much attention due to the increasing awareness of energy conservation and environmental problems. Electric vehicles (EV) are mobile energy storing systems, which can participate in energy trading. EV filling stations [1], which are a burden for low voltage (LV) networks [1], should be connected to medium voltage (MV) grids. EVs with vehicle-to-grid (V2G) capability are charged or discharged through substations.

Several papers have addressed microgrid architectures [2], [3] and bidirectional power converter topologies [4], [5], [6]. However, analysis covering utilization of several EVs as energy resources for peak shaving of utility networks loads is scarce. Conventional distribution substations [7] are either unidirectional in energy transfer or for energy storing purposes [8], [9]. These types of substations are not suitable for automated bidirectional energy exchange between EVs and utility networks. This paper presents a distribution substation topology with an integrated AC and DC bus, which is capable of charging EVs and utilizing EVs for peak shaving purposes. The paper analyzes power converter topologies for discharging of EVs up to the MV level. Different operation modes for peak shaving of utility network loads are simulated with MATLAB Simulink. The aim of the paper is to analyze peak shaving possibilities with EVs. Results of the analysis will be used for the development of control algorithms for the construction of a microgrid prototype. The paper is divided into two main parts. The first part describes the topology of the proposed substation and power converter topologies. The second part presents the simulation results of bidirectional energy exchange between EVs, battery energy storage units (BESU) and the utility networks with MATLAB Simulink.

\section{TOPOLOGY OF A DistribUTION SUBSTATION WITH AN INTEGRATED AC AND DC BUS}

Transformer substations are a part of the electric power system, concentrated in a given place to transmit electric energy, distribute power and step up or down the voltage. Substations for medium voltage grids (typically 6-24 kV) transform 3-phase MV to 3-phase LV (typically $400 \mathrm{~V} \mathrm{AC).}$

An example of a distribution substation topology for microgrids is presented in Fig. 1. The substation consists of a MV switchgear, transformers and several low voltage switchgears. The substation allows bidirectional energy exchange between EVs and consumers. Substation topology is implemented with an integrated $\mathrm{AC}$ and $\mathrm{DC}$ bus. Electric vehicles are connected with a common DC bus behind one AC-DC power converter [1]. For every EV in the common DC bus there are separate DC-DC converter subsystems, which consist of bidirectional DC-DC converters, protection and switching apparatuses. EVs are supplied from transformer T1 and consumers in the same substation are supplied from transformer T2. Bus coupler switch $\mathrm{BC} \_\mathrm{LV}$ separates the common AC bus for discharging EVs to the MV level. EVs can be discharged to the common $\mathrm{AC}$ bus if the main breaker LVA is opened and the bus coupler switch BC_LV is closed.

The battery energy storage units in the substations serve several purposes. In Fig. 1 BESUs are connected to the common AC bus. For every BESU in the common AC bus there are separate AC-DC-DC converter subsystems consisting of bidirectional AC-DC-DC converters, protection and switching apparatuses. BESUs can support either the LV side of EV supply or the LV side of consumers. For EV filling stations, the BESUs can be an additional buffer between the EVs and the utility network. BESUs are recharged during offpeak periods (e.g. night time). During peak periods BESUs can support fast charging of EVs, while drawing near-average power from the grid and reducing the series of charging peak loads for the utility network. In the case of a utility network power outage, the BESUs will ensure backup power capability to power consumer loads in an island mode. 


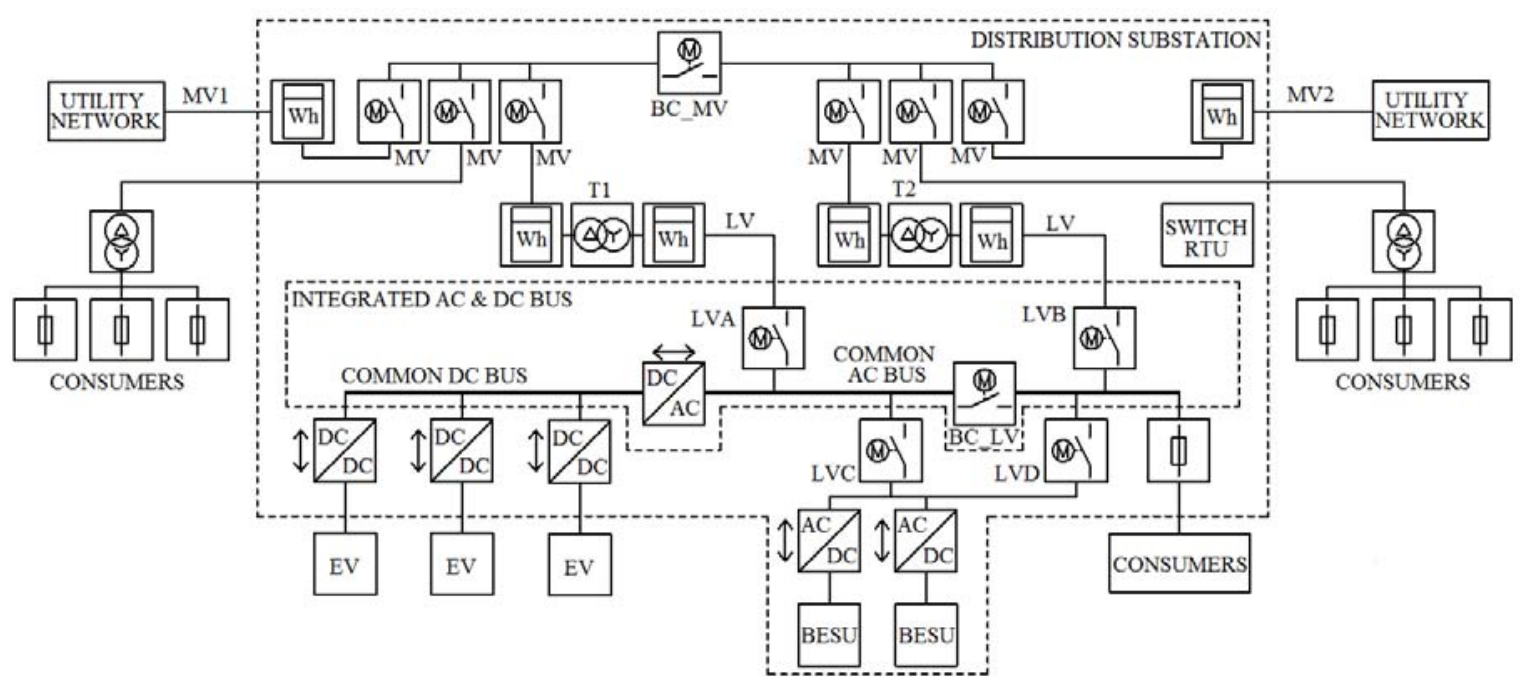

Fig. 1. Topology of a distribution substation with an integrated AC \& DC bus for microgrid applications.

As the number of renewable energy sources is increasing in the grid (e.g. wind and solar energy), the balancing of excess generation sources and load demands can be controlled through the substation. This enables stabilization of the grid voltage and frequency.

\section{BIDIRECTIONAL DC-DC POWER CONVERTER}

Bidirectional DC-DC converter controls charging or discharging current of a battery (by regulating output voltage). DC-DC converters have two main operating modes: buck mode (decreasing output voltage) and boost mode (increasing output voltage). In an integrated $\mathrm{AC}$ and $\mathrm{DC}$ bus topology, energy can be transferred from the common DC bus to the EV battery (vehicle) with buck mode and from the EV battery to the common DC bus with boost mode. The DC-DC converter with galvanic isolation necessitates the use of a transformer [10]. The galvanic isolation separates battery side from the grid side for protection and EMC reduction purposes. Fig. 2 illustrates a bidirectional double-leg full bridge DC-DC power converter topology with galvanic isolation.

In buck operation (motoring mode): switches SA1, SA2 and SA3, SA4 are controlled together (Fig. 2). There is no need of any control on the secondary side. All secondary switches (SB1, SB2, SB3, SB4) should be off and only anti-parallel diodes conduct. The four stages for buck operation can be summed up in the following equation [6]:

$$
\left\{\begin{array}{l}
S A 1, S A 2: \text { on } \rightarrow v_{L}=\frac{N 2}{N 1} V_{d}-V_{0} \\
S A 1, S A 2, S A 3, S A 4: \text { off } \rightarrow v_{L}=-V_{0} \\
S A 3, S A 4: \text { on } \rightarrow v_{L}=\frac{N 2}{N 1} V_{d}-V_{0} \\
S A 1, S A 2, S A 3, S A 4: \text { off } \rightarrow v_{L}=-V_{0}
\end{array}\right.
$$

where $v_{L}$ is the inductor voltage $(\mathrm{V}), V_{d}$ is the common DC bus side voltage $(\mathrm{V}), V_{0}$ is the battery (load) side voltage $(\mathrm{V}), \mathrm{N} 1$ is the number of transformer turns in the primary side, and $N 2$ is the number of transformer turns in the secondary side. The inductor ripple current $\Delta I_{L}$ and output voltage $\Delta V_{0}{ }_{C}$ ripple can be derived as [6]:

$$
\begin{gathered}
\Delta I_{L}=\frac{\Delta_{t} \cdot T_{s}}{L} \cdot V_{0}=\frac{N 2}{N 1} \cdot \frac{\left(D-2 D^{2}\right)}{L \cdot f_{s}} \cdot V_{d}, \\
\Delta V_{0_{-} C}=\frac{(0.5-D) \cdot V_{0}}{16 \cdot f_{s}^{2} \cdot L \cdot C_{P}},
\end{gathered}
$$

where $\Delta_{t}$ is the off time of switches (s), $T_{s}$ is the switching period of switches (s), $f_{s}$ is the switching frequency $(\mathrm{Hz}), D$ is the duty cycle of switches, $L$ is the inductance of the inductor $(\mathrm{H})$ and $C_{P}$ is the capacitance of capacitor $(\mathrm{F})$.

Simulations are based on the following parameters: $\mathrm{V}_{\mathrm{d}}=800 \mathrm{~V}, \mathrm{~V}_{0 \_ \text {min }}=300 \mathrm{~V}, \mathrm{~V}_{0 \_ \text {max }}=600 \mathrm{~V}$, transformer ratio $800 / 600 \mathrm{~V}(4 / 3), \quad \mathrm{D}=25 \%, \quad \mathrm{f}_{\mathrm{s}}=20 \mathrm{kHz}, \quad \Delta \mathrm{V}_{0 \mathrm{C}}=0.1 \mathrm{~V}$ and $\Delta \mathrm{I}_{\mathrm{L}}=0.1$ A. Using equations (2) and (3), $\mathrm{L}=37.5 \mathrm{mH}$ and $\mathrm{C}=3.1 \mu \mathrm{F} \approx 5 \mu \mathrm{F}$ can be deduced. For simplification, an isolating transformer is considered as an ideal kind.

To adjust the output voltage in a wide range (from $300 \mathrm{~V}$ to $600 \mathrm{~V}$ ) in buck mode, switches SA1 and SA3 operate as additional rectifiers.

Fig. 3 illustrates simulation results of the bidirectional DCDC converter in buck mode with MATLAB Simulink. $800 \mathrm{~V}$ DC supply is connected to the common DC bus side and different resistors are connected to the load side (3 $\Omega$ for $300 \mathrm{~V}, 4 \Omega$ for $400 \mathrm{~V}, 5 \Omega$ for $500 \mathrm{~V}, 100$ A output current in all cases). Different output voltages are compared in Fig. 3.

In boost operation (generation mode): switches SB1, SB2 and SB3, SB4 are controlled together (Fig. 2). There is no need of any control in the primary side. All primary switches (SA1, SA2, SA3, SA4) should be off and only anti-parallel diodes conduct. The switching period of switches can be divided into four stages. In the first stage, SB1 and SB2 conduct in the secondary side. In the second stage, all switches (SB1, SB2, SB3, SB4) have to be on to make a path for inductor current, this charges the inductor and the current increases linearly. 


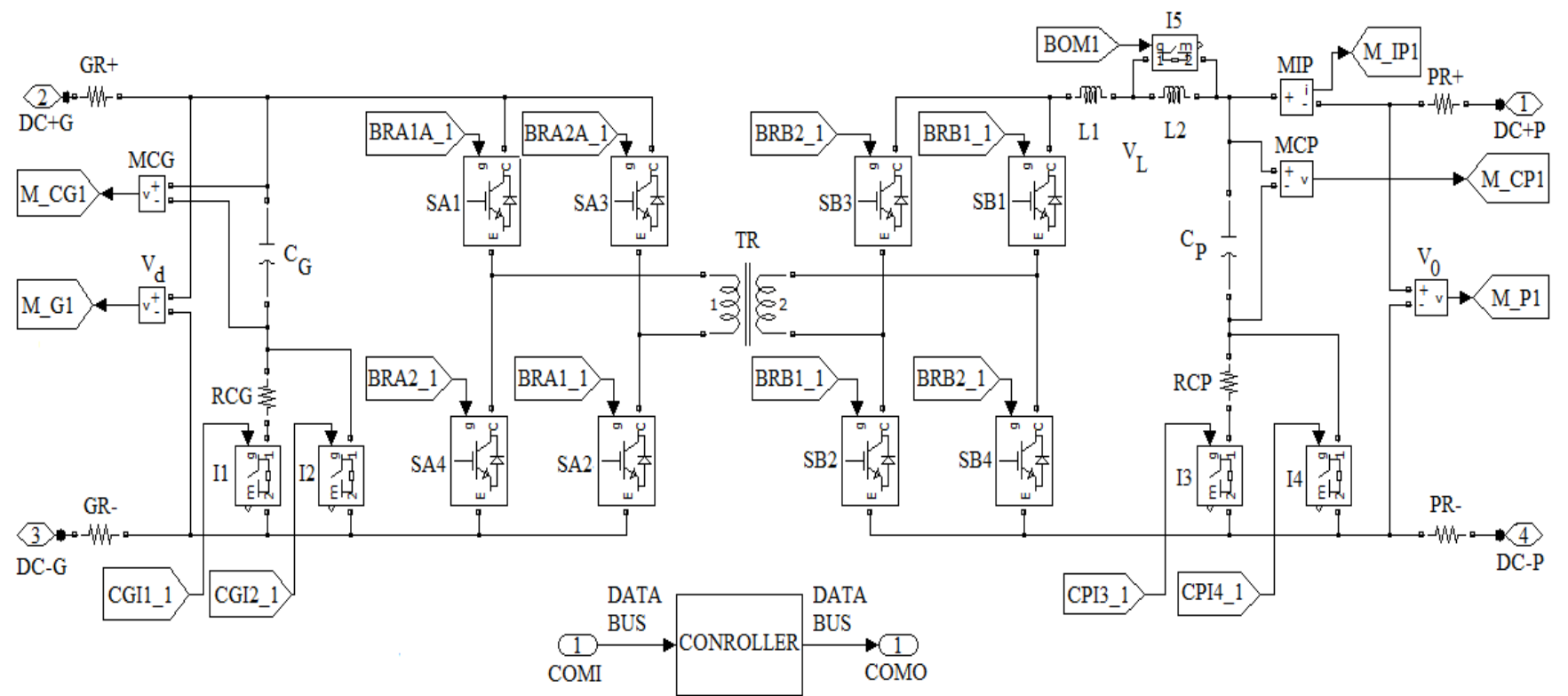

Fig. 2. MATLAB Simulink model of a bidirectional DC-DC power converter.

In the third stage, SB3 and SB4 conduct in the secondary side. In the fourth stage, all switches have to be on to make path for the inductor current. The four stages for boost operation can be summed up in the following equation [6]:

$$
\left\{\begin{array}{l}
S B 1, S B 2: \text { on } \rightarrow v_{L}=V_{0}-\frac{N 1}{N 2} V_{d} \\
S B 1, S B 2, S B 3, S B 4: \text { on } \rightarrow v_{L}=V_{0} \\
S B 3, S B 4: \text { on } \rightarrow v_{L}=V_{0}-\frac{N 1}{N 2} V_{d} \\
S B 1, S B 2, S B 3, S B 4: \text { on } \rightarrow v_{L}=V_{0}
\end{array} .\right.
$$

For continuous conditions, ripple current, as the function of $D$ can be derived as [6]:

$$
\Delta I_{L}(D)=\frac{N 1}{N 2} \cdot V_{0} \cdot(2 D-1) \cdot(1-D) \cdot \frac{1}{L \cdot f_{s}} .
$$

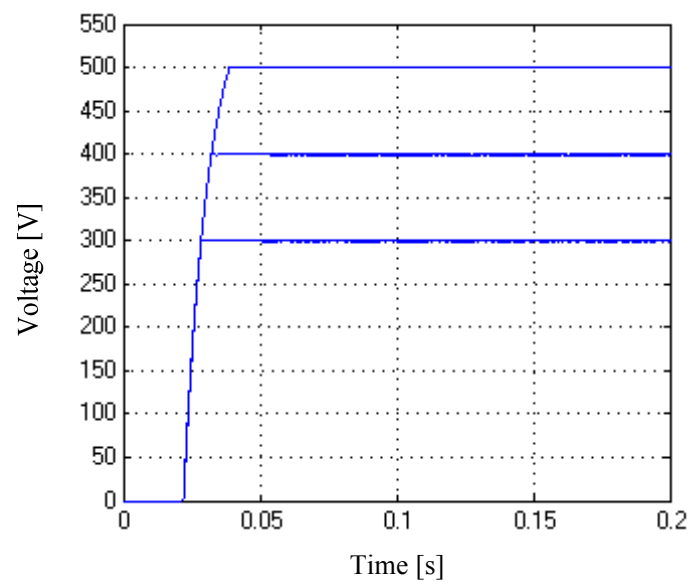

Fig. 3. Different simulated output voltages of the bidirectional DC-DC power converter in the MATLAB Simulink model in buck mode.
The relatively large inductor $(37.5 \mathrm{mH})$, which is necessary for low inductor ripple current in buck mode, causes instability in controlling of the common DC bus side voltage in boost mode. For this reason the inductor is split into two parts $\mathrm{L} 1(3.75 \mathrm{mH})$ and $\mathrm{L} 2(33.75 \mathrm{mH})$. In boost mode the $\mathrm{L} 2$ part is bypassed. A capacitor for the common DC bus side is chosen $\mathrm{C}_{\mathrm{G}}=1000 \mu \mathrm{F}$. Precharging resistors $2 \Omega$ (RCG, RCP) avoid sudden current peaks when connecting voltage to empty capacitors in either side.

\section{Bidirectional AC-DC POWer CONVERTER}

Bidirectional AC-DC converter rectifies $\mathrm{AC}$ voltage to $\mathrm{DC}$ voltage or inverts $\mathrm{DC}$ voltage to $\mathrm{AC}$ voltage. In an integrated $\mathrm{AC}$ and $\mathrm{DC}$ bus topology, AC-DC converter can be used to transfer energy from the common $\mathrm{AC}$ bus to the common $\mathrm{DC}$ bus or vice versa. Bidirectional AC-DC-DC converters have the AC-DC and the DC-DC power converters in series [4].

A three-level neutral point clamped (NPC) voltage sourced converter (VSC) [11], [12] was chosen in this paper as a bidirectional AC-DC converter with IGBTs/Diodes. The phase locked loop (PLL) shown in Fig. 4 is used to synchronize the AC-DC converter with the line voltage and also to calculate the transformation angle used in the $d-q$ transformation [13]. The PLL block measures the system frequency and provides the phase synchronous angle $\theta$ for the $d-q$ transformation unit.

Control of the converter is based on coordinate transformation between the stationary $\alpha-\beta$ and the synchronous rotating $d-q$ reference system [13]. For transferring energy over the voltage transformer in inverter mode, the Clark transformations for converting line voltages and currents from natural $a b c$ quantities to the $\alpha-\beta$ reference system have to be adjusted according to the transformer type. These can be presented for a star-star (Y/Y) transformer (or for transferring energy to the common $\mathrm{AC}$ bus without any transformer) for voltages (the same principle is applied for currents as well) [12]: 


$$
\left[\begin{array}{l}
v_{\alpha} \\
v_{\beta} \\
0
\end{array}\right]=\frac{2}{3}\left[\begin{array}{ccc}
1 & -\frac{1}{2} & -\frac{1}{2} \\
0 & \frac{\sqrt{3}}{2} & -\frac{\sqrt{3}}{2} \\
\frac{1}{2} & \frac{1}{2} & \frac{1}{2}
\end{array}\right]\left[\begin{array}{l}
v_{a} \\
v_{b} \\
v_{c}
\end{array}\right] .
$$

Delta-star (Dyn11) transformers are the most common in utility networks as delta side nullifies 3rd harmonics. Equation (6) can be written for Dyn11 transformers (where star side voltage is leading by $\pi / 6$ degrees) as [12]:

$$
\left[\begin{array}{l}
v_{\alpha} \\
v_{\beta} \\
0
\end{array}\right]=\frac{2}{3}\left[\begin{array}{ccc}
\frac{\sqrt{3}}{2} & -\frac{\sqrt{3}}{2} & 0 \\
\frac{1}{2} & \frac{1}{2} & -1 \\
0 & 0 & 0
\end{array}\right]\left[\begin{array}{l}
v_{a} \\
v_{b} \\
v_{c}
\end{array}\right]
$$

The active power or the DC voltage is controlled by the control of phase shift between $U_{L}$ (sinusoidal AC voltage in the AC network) and $U_{V}$ (fundamental line to line voltage on the valve side, Fig. 4). Reactive power is controlled by the control of the modulation index $(m)$. The instantaneous real and imaginary power of the inverter on the valve side can be expressed in terms of the $d q$ component of the current and the voltage on the valve side (Fig. 4) as follows [11]:

$$
\begin{gathered}
p=\frac{3}{2} \cdot \operatorname{Re}\left(u_{f}^{-d q} \cdot i_{v}^{-* d q}\right)=\frac{3}{2} \cdot\left(u_{f d} \cdot i_{v d}+u_{f q} \cdot i_{v q}\right), \\
q=\frac{3}{2} \cdot \operatorname{Im}\left(u_{f}^{-d q} \cdot i_{v}^{-* d q}\right)=\frac{3}{2} \cdot\left(-u_{f d} \cdot i_{v q}+u_{f q} \cdot i_{v d}\right) .
\end{gathered}
$$

If the reference of the $d q$-frame is selected such that the quadrature component of the voltage is very small and negligible, then equations (8) and (9) indicate that the active and the reactive power are proportional to the $d$ and $q$ component of the current, respectively. Accordingly, it is possible to control the active power (or the DC voltage or the DC current) and the reactive power (or the AC bus voltage) by the control of the current components $i_{v d}$ and $i_{v q}$, respectively.

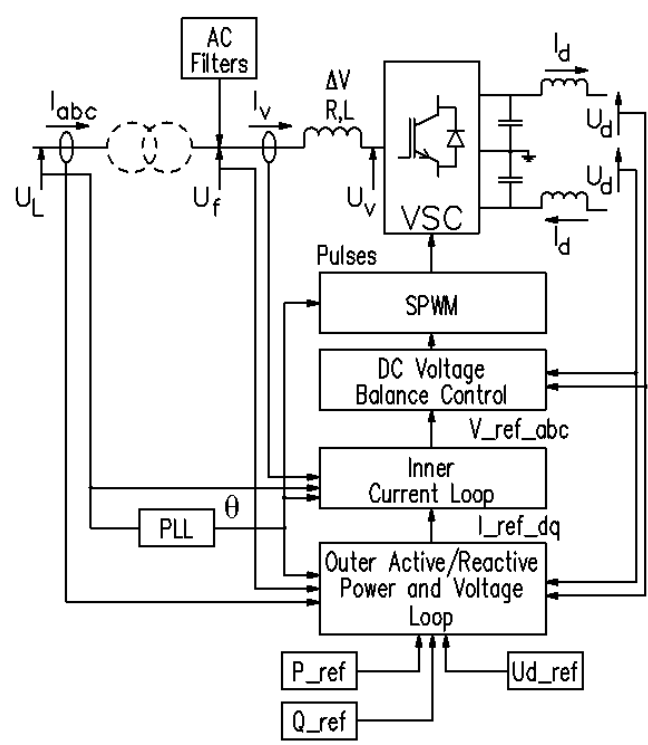

Fig. 4. Overview diagram of the VSC control system.

The active and reactive power and voltage loop contains the outer loop regulators that calculate the reference value of the converter current vector $\left(I_{d q}^{*}\right)$, which is the input to the inner current loop. Figure 4 shows a bidirectional AC-DC power converter topology that is similar to the topology simulated using the MATLAB Simulink model (Fig. 5).

\section{V.PEAK SHAVING}

The peak shaving application is particularly attractive for utility networks. Often industrial customers run apparatuses and devices that require significant amounts of power over relatively short time intervals during a day. EVs or BESUs can be used to reduce peak demand by discharging stored energy during load peaks (Fig. 6). For describing a load peak a reference value [8] is required $\left(P_{\text {Limit }}\right)$. The load peaks on the load curves are defined as the area above the reference value (peaking generation in Fig. 6). The required capacity $E_{c a p}$ of EVs or BESUs for peak shaving can be visualized as the area of the peak demand graph $y=P_{p w r}(t)$, where $P_{p w r}$ is the required power at time $t$ and $\Delta T$ is the required period for discharging. The area of peak generation in Fig. 6 is:

$$
E_{\text {cap }}(\Delta T)=\int_{t_{1}}^{t_{2}} P_{p w r}(t) d t-P_{\text {Limit }} \cdot \Delta T .
$$

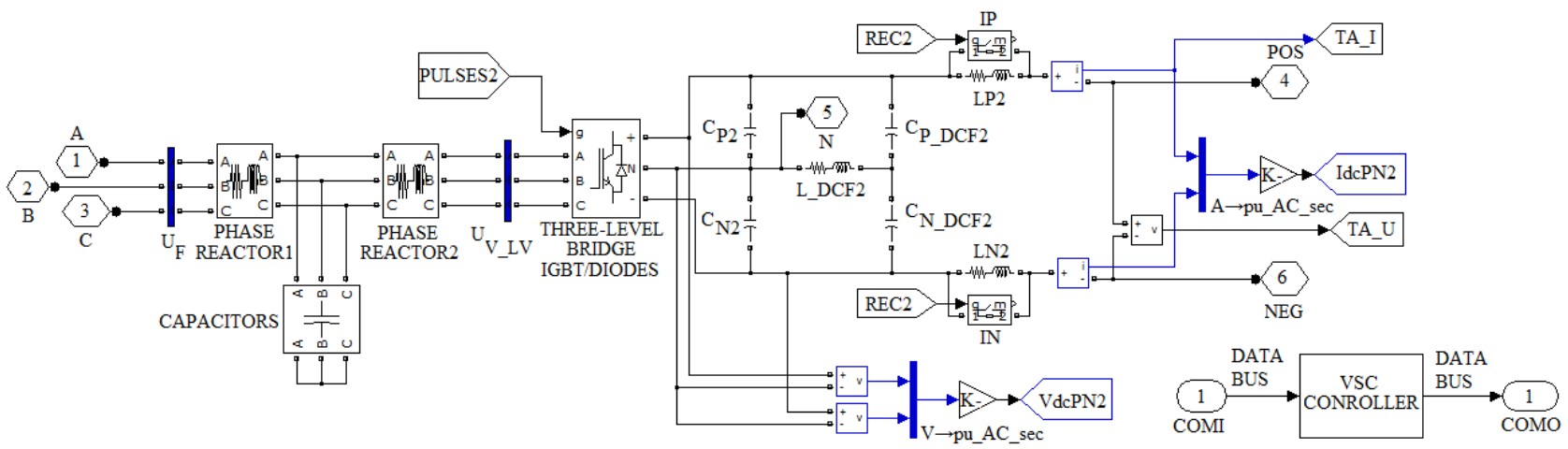

Fig. 5. MATLAB Simulink model of a bidirectional AC-DC power converter. 


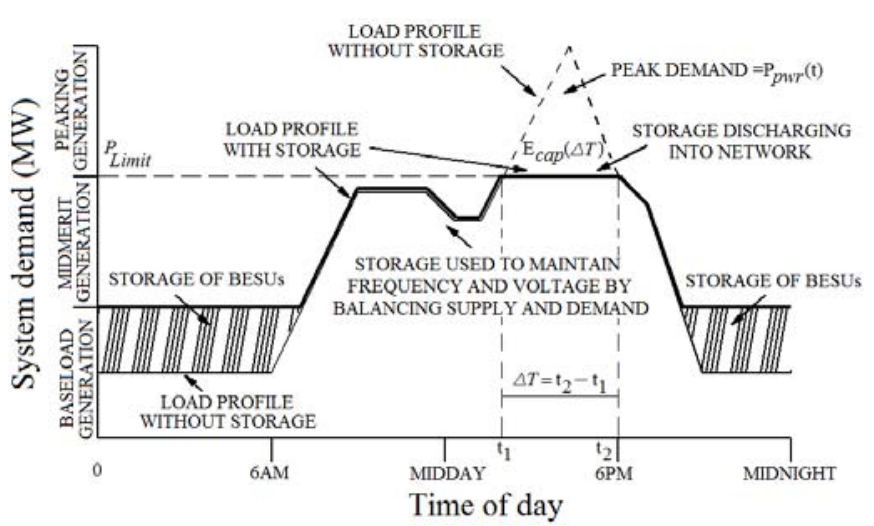

Fig. 6. Load profile of a utility network.

\section{Simulation Model of A Distribution SubStation}

Figure 1 shows a distribution substation topology (IT earthing system) that is similar to the topology simulated using the MATLAB Simulink model (Fig. 7). The model consists of two independent $24 \mathrm{kV}$ utility network supplies for redundancy, which are connected to the examined substation's MV switchgear. MV switchgear distributes MV voltage to the examined substation's low voltage sides (for EVs and consumers), and also to a nearby second distribution substation. The substation includes $250 \mathrm{kVA}$ voltage transformers 24/0.4 kV (TRANSF_A, TRANSF_B); smart meters before and after voltage transformers (SMA 1 , SMA2, SMB1, SMB2); low voltage circuit breakers (LVA, LVB, LVC, LVD); bus coupling switches (BC_LV, BC_MV); $50 \mathrm{~kW}$ DC-DC power converters (DCCS1-DCCS3) at the common DC bus for EVs (with nominal voltage of $330 \mathrm{~V}$ DC); a $150 \mathrm{~kW}$ AC-DC power converter (ACDCS1) between the common DC bus and the common $\mathrm{AC}$ bus; $50 \mathrm{~kW}$
AC-DC-DC power converters (ACDCS2, ACDCS3) for BESUs (with a nominal voltage of $460 \mathrm{~V}$ DC).

Four simulations were analyzed as follows: firstly, fast charging of EVs without any peak shaving; secondly, fast charging of EVs with peak shaving by the BESUs; thirdly, discharging of EVs to the common AC bus for utility load peak shaving; fourthly, discharging of EVs to medium voltage side for utility load peak shaving (voltage transformers and current transformers from the MV side provide input for ACDCS1). This study examines only active power let-through for the utility network. Medium voltage transformers are simulated as ideal transformers. Reactive and apparent power let-through will be analyzed in future papers.

The maximum active power let-through for the utility was chosen $600 \mathrm{~kW}$ in the simulations. The second distribution substation required $400 \mathrm{~kW}$ of active power for consumers in all four simulations. The substation examined required $100 \mathrm{~kW}$ up to $250 \mathrm{~kW}$ of active power for consumers. Additionally, charging of EVs required $150 \mathrm{~kW}$ of active power. All maximum required powers added together result in $800 \mathrm{~kW}$ of active power. In the concept of Smart Grids [1] generation and consumption of energy should be in balance such that sufficient energy is available at all times and with high quality.

The purpose of this model is not to simulate a particular bidirectional AC-DC or DC-DC converter, but to evaluate bidirectional energy exchange possibilities between the EVs and the utility network for peak shaving of utility network loads. Total harmonic distortion (THD) values registered by smart meters in simulations are also presented: THD of the current of the utility network input $T H D_{I U T I}$ (at M_MV1); THD of the current of the common ac bus $T H D_{I_{-} S M A 2}$ (at SMA2).

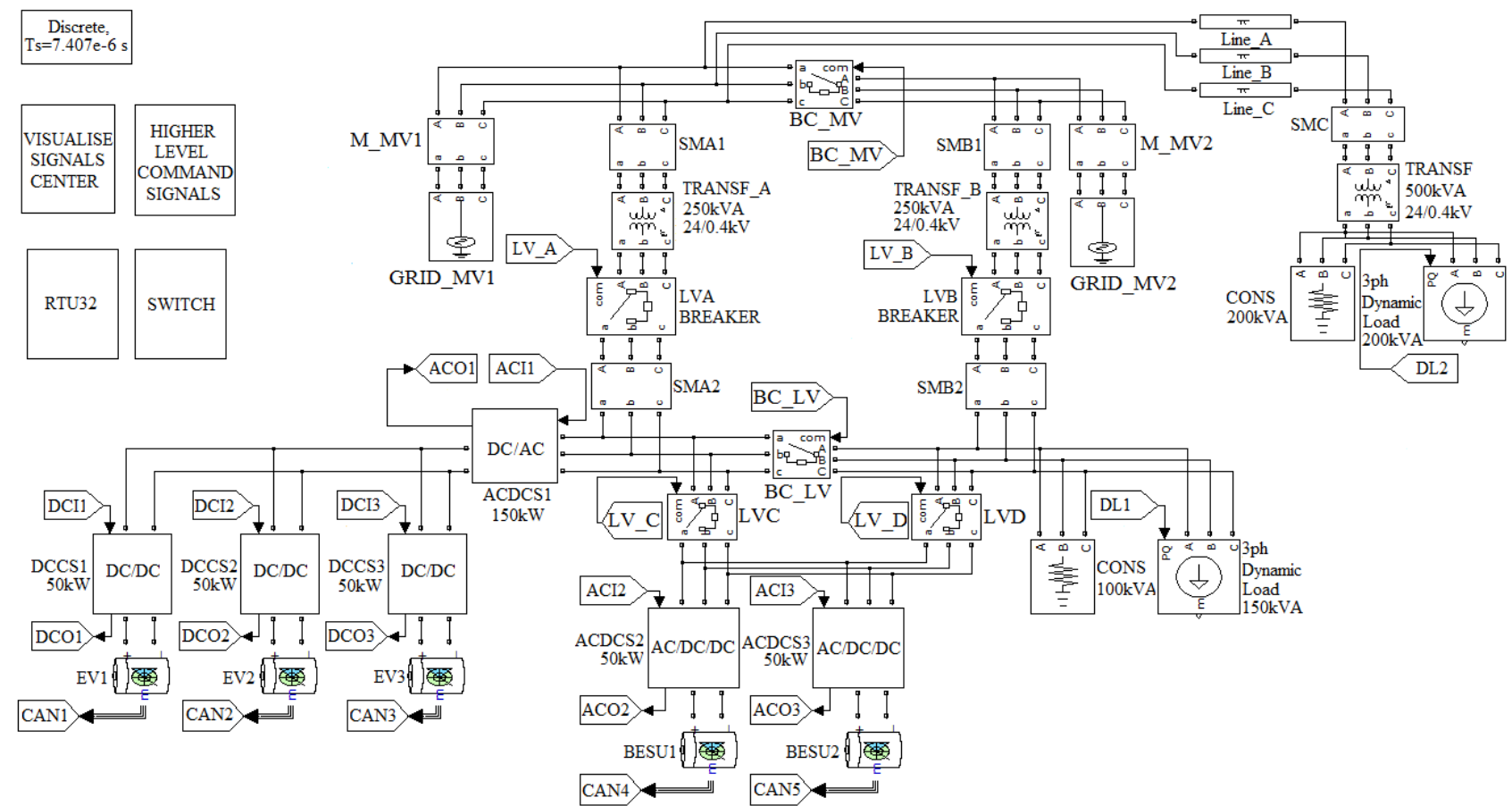

Fig. 7. MATLAB Simulink model of the distribution substation with an integrated AC \& DC bus, EVs and BESUs. 


\section{Simulation Results}

Firstly, fast charging of EVs without any peak shaving was simulated. In all four simulations, second utility network supply was considered as unavailable and all the loads had fallen for the first utility network supply. Circuit breaker LVA and bus coupling breaker BC_LV were closed. Circuit breakers LVB, LVC and LVD were open. Examined substation was loaded with $100 \mathrm{~kW}$ of consumers (total of $500 \mathrm{~kW}$ for the utility network with the second substation and without EVs). All three EV charging stations were loaded simultaneously at $0.22 \mathrm{~s}$ (fast charging with $100 \mathrm{~A}$ and $36 \mathrm{~kW}$ ). The results from the simulated model are presented in Fig. 8. Figure 8 illustrates the utility load, the state of charge and the current of the EV1 in the MATLAB Simulink model at the consumer stage of the EVs. The maximum load for the utility network was exceeded by $3 \%$. THD $_{\text {I_UT1 }}<0.1 \%$ and $\mathrm{THD}_{\text {I SMA2 }}<0.1 \%$ at the continuous operation of the AC-DC power converter ACDCS1.

Secondly, fast charging of EVs with peak shaving by the BESUs was examined. Circuit breakers LVA, LVC and bus coupling breaker BC_LV were closed. Circuit breakers LVB and LVD were open. Examined substation was loaded with $100 \mathrm{~kW}$ of consumers (total of $500 \mathrm{~kW}$ for the utility network). All three EV charging stations were loaded simultaneously at $0.22 \mathrm{~s}$ (fast charging with $100 \mathrm{~A}$ and $36 \mathrm{~kW}$ ). Energy stored in both BESUs were discharged to the common AC bus simultaneously at $0.1 \mathrm{~s}$ (target value of $50 \mathrm{~kW}$ for each BESU). The results from the simulated model are presented in Fig. 9. Figure 9 illustrates the utility load, the state of charge and the current of the BESU1 in the MATLAB Simulink model at the consumer stage of the EVs. The maximum load for the utility was not exceeded. The discharging of BESUs in parallel with fast charging of EVs reduces load for the utility network. $\mathrm{THD}_{\mathrm{IUT1}}<0.1 \%$ and $\mathrm{THD}_{\mathrm{I}_{-} \mathrm{SMA} 2}<0.3 \%$ at continuous operation of ACDCS 1 .

Thirdly, discharging of EVs to the common AC bus for the utility load peak shaving was examined. Circuit breaker LVA and bus coupling breaker BC_LV were closed. Circuit breakers LVB, LVC and LVD were open. The substation examined required $250 \mathrm{~kW}$ of active power for consumers and $400 \mathrm{~kW}$ of active power was required by the second distribution substation. The load in the examined substation was ramped up linearly, as presented in Fig. 10. All three EV charging stations were set to discharge mode simultaneously with the start of the simulation. The target for the AC-DC converter 1 is to utilize the full power of EVs (total of $150 \mathrm{~kW}$ ). The results from the simulated model are presented in Fig. 10. Figure 10 illustrates the utility load, ramping of dynamic load 1 and the state of charge of the EV1 in the MATLAB Simulink model at the producer stage of the EVs. The maximum load for the utility was not exceeded. $\mathrm{THD}_{\text {I_UT1 }}<0.1 \%$ and $\mathrm{THD}_{\mathrm{I}_{\text {ISMA2 }}}<0.4 \%$ at the continuous operation of ACDCS1.

Fourthly, discharging of EVs to a medium voltage side for utility load peak shaving was examined. Circuit breakers LVA, LVB and bus coupling breaker BC_MV were closed. Circuit breakers LVC, LVD and bus coupling breaker BC_LV were open. The examined substation required $250 \mathrm{~kW}$ of active power for consumers and $400 \mathrm{~kW}$ of active power was required by the second distribution substation. The load in the examined substation was also ramped up linearly, as presented in the third simulation. All three EV charging stations were set to discharge mode simultaneously with the start of the simulation.
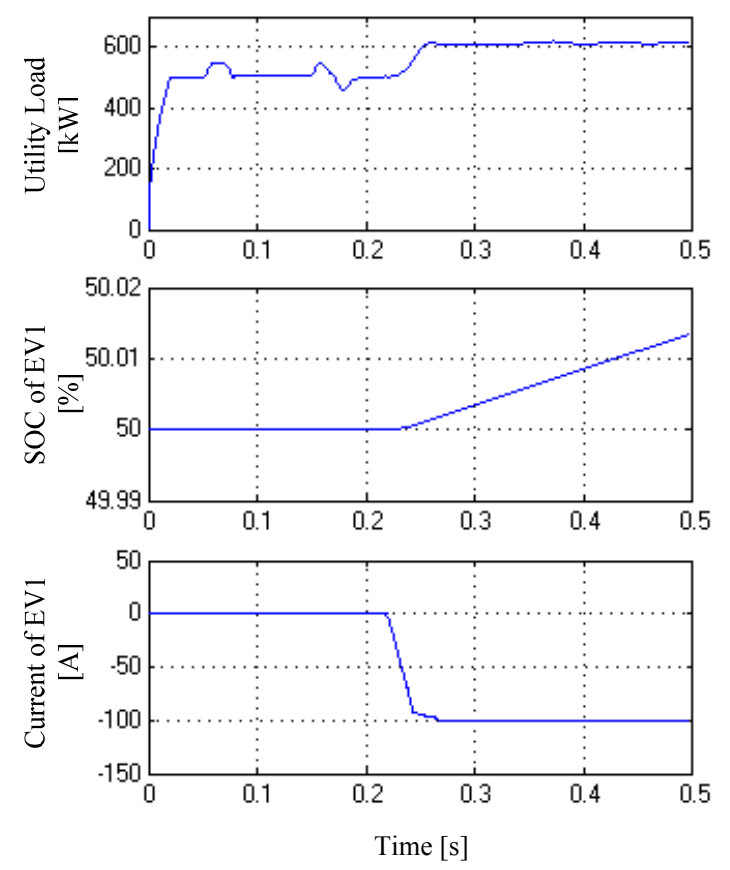

Fig. 8. Utility load, state of charge and current of the EV1 in the MATLAB Simulink model at the consumer stage of the EVs without peak shaving.
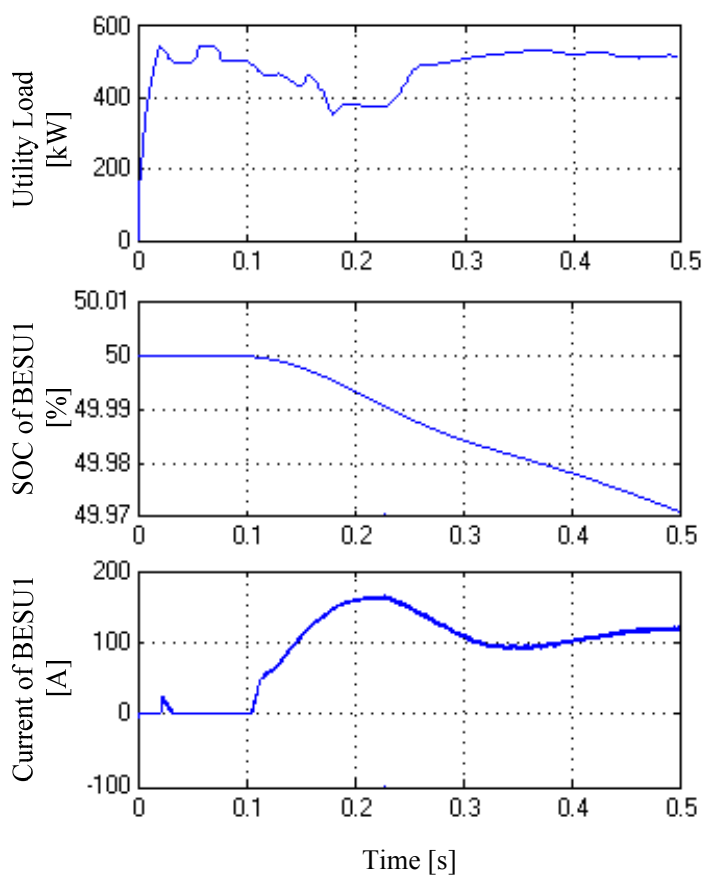

Fig. 9. Utility load, state of charge and current of the BESU1 in the MATLAB Simulink model at the consumer stage of the EVs with peak shaving by the BESUs. 

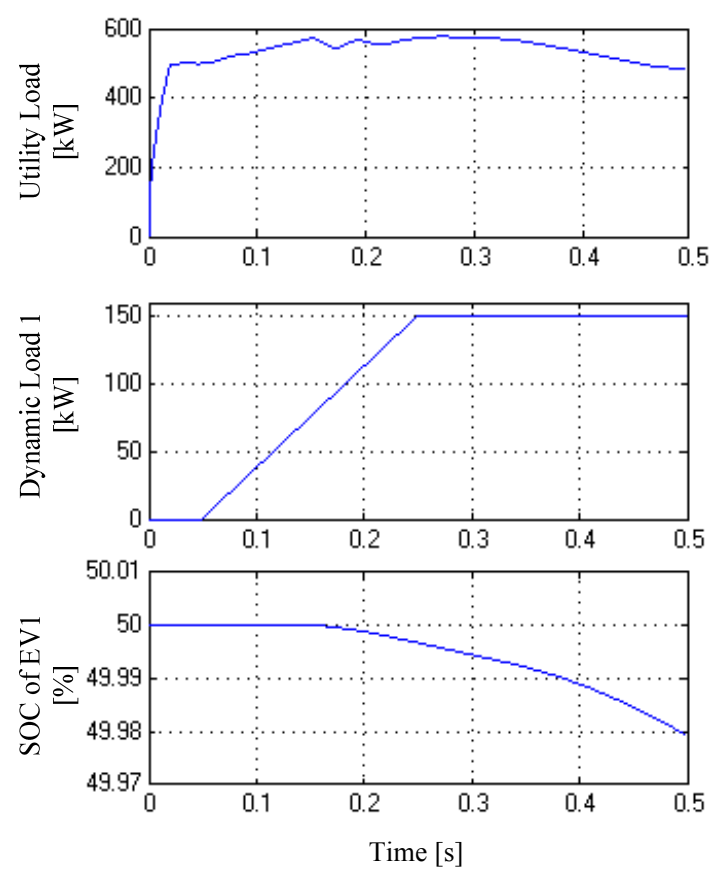

Fig. 10. Utility load, ramping of dynamic load 1 and state of charge of the EV1 in the MATLAB Simulink model at the producer stage of the EVs for utility load peak shaving at the common AC bus.
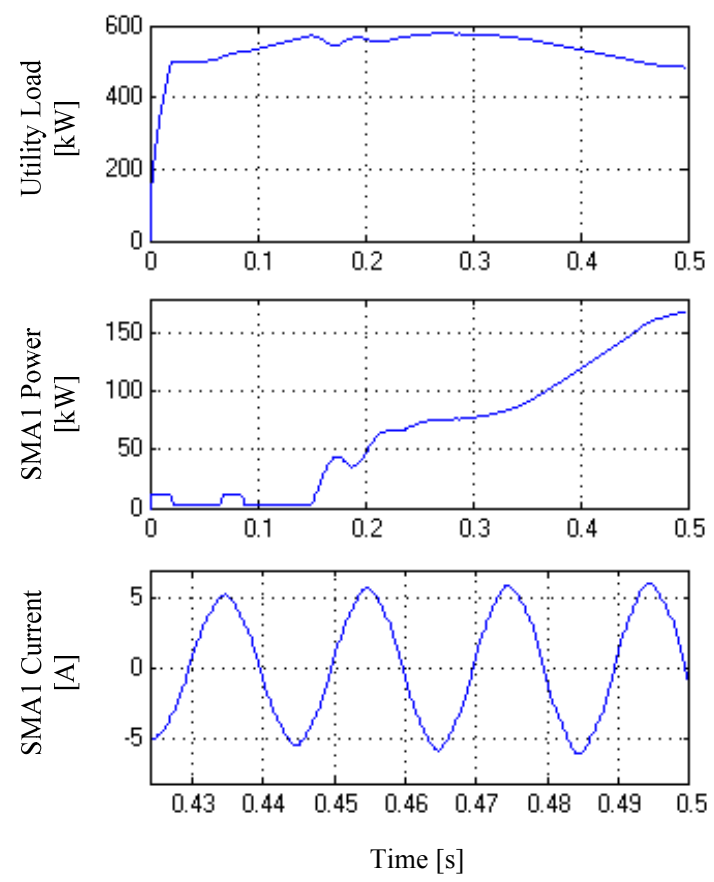

Fig. 11. Utility load, transferred active power to MV side and current recorded by smart meter SMA1 in the MATLAB Simulink model at the producer stage of the EVs for utility load peak shaving at the MV level.

The target for AC-DC converter 1 is to utilize the full power of EVs (total of $150 \mathrm{~kW}$ ). The results from the simulated model are presented in Fig. 11. Figure 11 illustrates the utility load, the transferred active power to the MV side and the current recorded by the smart meter SMA1 in the MATLAB Simulink model. The maximum load for the utility was not exceeded. $\mathrm{THD}_{\text {I_UT } 1}<0.1 \%$ and $\mathrm{THD}_{\text {I_SMA } 2}<0.2 \%$ at the continuous operation of the AC-DC power converter ACDCS1.

\section{VIII.CAPACITY OF EVS FOR PEAK SHAVING}

Battery electric vehicles are becoming more and more attractive with the advancement of Li-ion battery technology. Many car manufacturers have developed their own versions of EVs. E.g. Mitsubishi i-MiEV [14] and Citroën C-Zero have a $16 \mathrm{kWh} 50 \mathrm{Ah} \mathrm{Li-ion}$ battery pack with nominal voltage of $330 \mathrm{~V}$. If these EVs are discharged at $2 \mathrm{C}$ rate $(100 \mathrm{~A})$, the output power from the EV battery is $33 \mathrm{~kW}$. Considering efficiencies of power converters (e.g. $95 \%$ for the bidirectional DC-DC power converter [6], $96 \%$ for the bidirectional AC-DC power converter [15]) and discharge efficiency of EV Li-ion battery pack (93\% [16]), maximum of $28 \mathrm{~kW}$ of active power can be extracted from one EV to the common AC bus (Fig. 1) of LV side (total combined efficiency of $85 \%$ ). Electric vehicle owners prefer that their $\mathrm{EV}$ batteries are discharged as little as possible as every discharge cycle degrades the batteries. Considering depth of discharge (DOD) of $20 \%(3.2 \mathrm{kWh}), 28 \mathrm{~kW}$ of active power to the LV side can be extracted from one EV for 7 minutes. The more EVs are connected to the common DC bus (Fig. 1) the more active power can be extracted.

In microgrid applications distribution substation can be viewed as an energy router and it is the function of the substation's main controller in the higher level to determine when to utilize EVs or BESUs for peak shaving. In the Fig. 1 topology it is considered that EVs are discharged when fixed amount of active power is ordered from the utility network (MV level) or from the consumers side (LV level). BESUs are discharged only to the LV level for the support of fast charging of EVs or to ensure backup power capability to power consumer loads in the case of a utility network power outage. The discharging of EVs or BESUs is controlled through low level control of power electronic converters.

\section{FUTURE STUDIES}

Tallinn University of Technology is constructing an experimental microgrid that enables us to study energy flows and data communication during EV charging. The microgrid consists of a fast charging station for EV charging and a battery pack for storing electric energy. The basic functions and operation modes (including protection algorithms), such as energy transmission from the power grid to the energy storing system, EV battery charging, balancing power loads and other functions have to be developed, tested and analyzed. Management and control algorithms for the microgrid controller have to be fine-tuned. During experimentation, data values will be collected for further analysis. Primary goals in the construction of the microgrid are to analyze the quality of energy flow, efficiency and harmonic levels during EV charging through the microgrid and electromagnetic compatibility related issues. The analysis will indicate needs for modifications to be made in the microgrid structure to optimize and improve the overall efficiency and power factor levels in the system to ensure the quality of electricity in 
accordance with international standards. Practical applications will show possible drawback areas in the communication between the devices, which will then have to be solved with different control. Future studies will focus more on V2G and microgrid, as well as will address possibilities to transfer energy to the common AC bus or to the power grid for peak shaving of loads with synchronization related issues. Results from microgrid experiments will be published in future papers.

\section{X.CONCLUSIONS}

This paper has reviewed a distribution substation topology with an integrated AC and DC bus, which is capable of charging EVs and utilizing EVs for peak shaving purposes. It has been proven through simulations that an integrated $\mathrm{AC}$ and DC bus is the main topology solution for integrating fast charging stations for EVs to power grids. Simulation results have verified that bidirectional energy exchange between the utility network and EVs can be used for peak shaving of utility networks loads. EVs can be discharged up to the MV level.

Utilization of BESUs with fast charging of EVs can effectively reduce the load for the utility network (Fig 9). The amount of support and duration required determines the sizing of BESUs. EVs can provide short term support either for the LV side (Fig 10) or for the MV side (Fig 11). Utilization of EVs for peak load shaving and demand response can reduce/postpone the need to make new investments into building new power sources or grids to meet peak demand.

The simulation model is suitable for the development of control algorithms for a fully automated substation, which can be used for peak shaving of utility network loads. The substation for microgrid applications should be ready to simultaneously serve AC and DC buses. This means that the core control program in its main controller should integrate control and protection functions for AC bus, common DC bus and the link from AC bus to the utility grid. Before constructing a real life substation, a smaller prototype has to be examined. First, the control functions will be tested in the experimental microgrid constructed at Tallinn University of Technology. Experiments with the microgrid will give vital data about the charging and discharging algorithms for EVs and communication between the devices. These studies make possible to construct a larger real life substation capable of supplying power to several EV charging stations and that will be a part of a microgrid or a module of Smart Grid solutions.

\section{ACKNOWLEDGMENT}

This research work has been supported by European Social Fund (project "Doctoral School of Energy and Geotechnology II"), Estonian Ministry of Education and Research (project SF0140016s11), Estonian Science Foundation (Grant ETF9350), Estonian Archimedes Foundation (project AR10126), SmartGrids Era-Net (project GERA1) and European Regional Fund.

\section{REFERENCES}

[1] M. Mägi, "Analysis of Distribution Substation Topologies for Energy Exchanging between EV and Utility Networks," in11th International Symposium "Topical Problems in the Field of Electrical and Power Engineering" Pärnu 2012, 2012, pp. 158-167.
[2] J.M. Guerrero, M. Chandorkar, T-L. Lee, P.C. Loh, "Advanced Control Architectures for Intelligent Microgrids - Part I: Decentralized and Hierarchical Control; Part II: Power Quality, Energy Storage, and AC/DC Microgrids," in Industrial Electronics, IEEE, issue 99, 2012, pp 18.

[3] M.Y. Nguyen, Y.T. Yoon, N.H. Choi, "Dynamic Programming Formulation of Microgrid Operation with Heat and Electricity Constraints," in Transmission \& Distribution Conference \& Exposition: Asia and Pacific, IEEE, 2009, pp 4.

[4] J.F. Zhao, J.G. Jiang, X.W. Yang, "AC-DC-DC Isolated Converter with Bidirectional Power Flow Capability" in IET Power Electron., Vol. 3, 1ss. 4, 2010, pp 472-479.

[5] G.Y. Choe, J-S. Kim, B-K. Lee, C-Y. Won, T-W. Lee, "A Bi-directional Battery Charger for Electric Vehicles Using Photovoltaic PCS Systems," in Vehicle Power and Propulsion Conference VPPC10, IEEE, 2010, pp 6.

[6] M. Erfani, "Design of a Bidirectional On-board Battery Charger in Hybrid Electric Vehicle Applications," Master of science thesis, Chalmers University of Technology, Göteborg, Sweden, 2011.

[7] M. Mägi, K.Peterson, and E.Pettai, "Analysis of Protection and Control Functions of Low Voltage Part of Substation for Smart Grid Applications," in "Proceedings of $8^{\text {th }}$ International Conference 2012 Electric Power Quality and Supply Reliability: 2012 Electric Power Quality and Supply Reliability” Tartu 2012, 2012, IEEE, pp. 297-304.

[8] A. Oudalov, R. Cherkaoui and A. Beguin, "Sizing and Optimal Operation of Battery Energy Storage System for Peak Shaving Application," in Power Tech, 2007 IEEE Lausanne , 2007, pp. 621-625.

[9] ABB, "Energy Storage Modules (ESM)," [Online]. Available: http://www.abb.com/product/db0003db004279/420d3ae414b13c128525 7782004792b5.aspx. [Accessed: March 1, 2013].

[10] IEC 60364-7-722, "Low Voltage Electrical Installations Part 7-722: Requirements for Special Installations or Locations - Supply of Electric Vehicle," 2012.

[11] K. Mohamed, Z.S. Ahmed, H. Samir, F.M. Karim, and A. Rabie, "Performance Analysis of a Voltage Source Converter (VSC) based HVDC Transmission System under Faulted Conditions," Leonardo Journal of Sciences ISSN 1583-0233, Issue 15 (July-December), pp. 3346, 2009.

[12] MathWorks, "VSC-Based HVDC Link," [Online]. Available: http://www.mathworks.se/help/physmod/powersys/ug/vsc-based-hvdclink.html. [Accessed: March 1, 2013].

[13] M. Bobrowska-Rafal, K. Rafal, G. Abad, and M. Jasinski, "Control of PWM Rectifier Under Grid Voltage Dips," Bulletin of the Polish Academy of Sciences, vol. 57, no. 4, pp. 7, 2009.

[14] M. Kamachi, H. Miyamoto, Y. Sano, "Development of Power Management System for Electric Vehicle "i-MiEV"," in Power Electronics Conference (IPEC) 2010, 2010, IEEE, pp 2949-2955.

[15] H. Makkonen, J. Partanen, P. Silventoinen, "Concept of Battery Charging and Discharging in Automotive Applications," in Power Electronics Electrical Drives Automation and Motion (SPEEDAM) 2010 International Symposium, 2010, IEEE, pp 1664-1669.

[16] M. Eberhard, M. Tarpenning, "The $21^{\text {st }}$ Century Electric Car," Tesla Motors Inc, July 2006, pp 10.

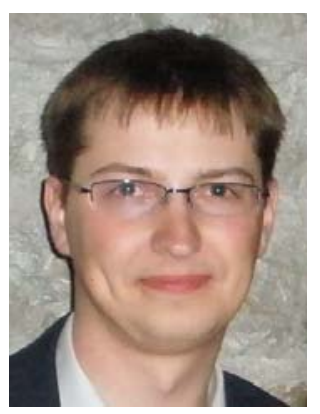

M. Mägi received the B.S. degree from Tallinn University of Technology (TUT) in 2004 and the M.Sc. degree from TUT in 2005. From 2011 he is a $\mathrm{PhD}$ student in the Department of Electrical Engineering of TUT, Tallinn, Estonia.

He has been working for 9 years as Electrical Engineer in an Estonian company AS Harju Elekter Elektrotehnika. The company manufactures distribution substations, MV and LV switchgears. His main scientific interests lie in the distribution substations, energy transfer between electric vehicles and utility network. He is an author or co-author of 7 publications.

E-mail: marek.magi@he.ee

Postal address: Faculty of Power Engineering, Tallinn University of Technology, Ehitajate tee 5, 19086 Tallinn, Estonia. 